effects of overtime work hours and exercise habits on psychological distress.

Methods We used data from a health examination of 1082 workers in FY 2013. We obtained information on working hours in the most recent month from the personnel records of the surveyed company. Overtime work hours per month were classified into three groups: short ( $<45$ hours), medium (45-79 hours), and long ( $\geq 80$ hours). Exercise habits were classified into two groups using a self-administered questionnaire: yes or no. Odds ratios (ORs) of psychological distress, defined as scores $\geq 4$ on the 12-item General Health Questionnaire, were calculated using multiple logistic regression analyses adjusting for age, gender, marital and residence status, occupation, drinking habits, smoking history and psychosocial work characteristics.

Results Compared to the short overtime with exercise habits group, the ORs (95\% confidence intervals) for psychological distress were significant for the medium overtime with exercise habits group $(\mathrm{OR}=1.81$ [1.20-2.75]), medium overtime without exercise habits group $(\mathrm{OR}=2.11$ [1.37-3.25]), and long overtime without exercise habits group $(\mathrm{OR}=3.03$ [1.64-5.58]). No significant ORs were observed for any other group combinations.

Discussion In the medium overtime group, overtime work hours were significantly associated with psychological distress regardless of exercise habits. However, in the long overtime group, this significant association disappeared among those with exercise habits. Our findings suggest that exercise habits reduce psychological distress in relation to long (i.e., $\geq 80$ hours) overtime work hours.

\section{WHICH STRESS-RELATED FACTORS AFFECT THE MENTAL HEALTH OF NUCLEAR EMERGENCY WORKERS OVER A LONG PERIOD?}

${ }^{1} \mathrm{H}$ Hiro*, ${ }^{1} \mathrm{~A}$ Hino, ${ }^{1} \mathrm{~K}$ Mafune, ${ }^{2} \mathrm{~A}$ Inoue, ${ }^{3} \mathrm{~J}$ Shigemura, ${ }^{4} \mathrm{M}$ Yamada, ${ }^{4} \mathrm{~T}$ Okubo. ${ }^{1}$ University of Occupational and Environmental Health, Japan, Kitakyushu, Japan; ${ }^{2}$ Kitasato University School of Medicine, Japan; ${ }^{3}$ National Defense Medical College; ${ }^{4}$ Radiation Effect esearch Foundation

\subsection{6/oemed-2018-ICOHabstracts. 1739}

Introduction Fukushima Daiichi Nuclear Power Plant suffered serious damage the Great East Japan Earthquake and Tsunami of 2011. This study examines the association between mental health status and stress-related factors in the nuclear emergency workers 4-5 years after the disaster.

Methods In total, 1572 workers completed a self-administered questionnaire by mail. Mental health status was measured by the K6. The stress-related factors we evaluated life events, stress coping, sense of coherence, self-esteem, self-efficacy, resilience, social support, life satisfaction, job satisfaction and slanderous defamation. Multiple regression analysis was performed with the $\mathrm{K} 6$ score as the explained valuable and stress-related factors as the explanation valuables.

Result The K6 score was significantly high in the workers who had worked for a long period of time. It was also related to the scores of life events, stress coping, self-esteem, life satisfaction, work satisfaction and slanderous defamation. The slanderous defamation score most strongly related to the K6 score.

Discussion Long term follow-up is for nuclear emergency workers who work for long period of time, and slanderous defamation is one of the most important measures support the mental health of these workers
898

DEVELOPING A CULTURALLY RELEVANT WORKPLACE MENTAL HEALTH E-HEALTH APPLICATION FOR THE CANADIAN INDIGENOUS POPULATION

1,2,3,4 VL Kristman*, ${ }^{5} \mathrm{~A}$ Gilbeau. 'Department of Health Sciences, Lakehead University, Thunder Bay, Ontario, Canada; ${ }^{2}$ Division of Human Sciences, Northern Ontario School of Medicine, Thunder Bay, Ontario, Canada; ${ }^{3}$ Institute for Work and Health, Toronto, Ontario, Canada; ${ }^{4}$ Dalla Lana School of Public Health, University of Toronto, Toronto, Ontario, Canada; ${ }^{5}$ Nokiiwin Tribal Council, Thunder Bay, Ontario, Canada

\subsection{6/oemed-2018-ICOHabstracts. 1740}

Introduction Workplace stress stemming from lateral violence in the workplace is problematic for Indigenous workers. Unlike workplace bullying, lateral violence differs due to the root cause. Canadian Indigenous people are abusing their own people in similar ways to how they have been abused. It is a cycle of abuse and its roots lie in factors of colonisation, oppression, intergenerational trauma and the ongoing experiences of racism and discrimination. Information on mental health and mental health resources within Indigenous workplaces is lacking. Therefore, our objective was to develop an ehealth intervention for Indigenous mental health in the workplace.

Methods We participated in a CIHR 'Work Stress and Wellbeing Hackathon' to achieve our objective. The Hackathon was a two and a half day event where participants work with an e-health team to devise a solution to their problem. The event was collaborative and involved multiple perspectives in the development of the e-mental health solution. In addition to the principal investigator and knowledge user, the team included an individual with lived experience and technical expertise, including designers and programmers.

Results At the end of the Hackathon we had developed a prototype of a working e-mental health application based on the medicine wheel and Indigenous cultural teachings. We also developed a relationship with an e-health company, Cossette Health, who will be working with us over the next year to turn the prototype into a viable working e-health application. We will demonstrate our working app at the conference.

Conclusion Future goals of the project will be to evaluate the effectiveness of the completed e-mental health application, in the communities affiliated with the Nokiiwin Tribal Council, with respect to improving access to relevant mental health information and reducing workplace lateral violence.

\section{TEACHER'S PREVALENCE OF DEPRESSIVE SYMPTOMS IN KOREA}

${ }^{1}$ YR Lee ${ }^{*},{ }^{1}$ HR Kim, ${ }^{1}$ SY Lee, ${ }^{2}$ HH Kim, ${ }^{2}$ WY Lee. ${ }^{1}$ Seoul St. Mary's Hospital of the Catholic University of Korea, Seoul, Republic of Korea; ${ }^{2}$ Chamgyoyook Research Institute, Seoul, Republic of Korea

\subsection{6/oemed-2018-ICOHabstracts. 1741}

Introduction Mental health problems of teachers have been raised due to increased workload, violence, emotional labour in students and parents response processes. The purpose of this study is to investigate the mental health status and to find way to improve teacher's working environment.

Methods Total 79 schools in 8 groups were randomly selected considering level(elementary, middle, academic high, vocational high) and area(urban, rural) using National Educational Statistics 2015. The questionnaire including CES-D was mailed to 
randomly selected schools and survey was conducted for all teachers in these schools during July to August 2016. Probable depression was defined as a CES-D score of 16 or more and definite depression was defined as a score of 25 or more.

Results 1617 out of total 3345 in 79 schools responded to the survey. Except for those who were missing information, 1123 teachers were included this analysis. Compared with the general population, teacher's prevalence of probable depression was higher in the $30 \mathrm{~s}$ and $40 \mathrm{~s}$ (28.99\% vs $22.29 \%, 26.46 \%$ vs $20.87 \%$, respectively) and definite depression was higher in the $20 \mathrm{~s}, 30 \mathrm{~s}$ and $40 \mathrm{~s}(15.38 \%$ vs $100.07 \%, 14.01 \%$ vs $8.08 \%, 10.46 \%$ vs $7.57 \%$, respectively). In the case of high school homeroom teacher, the 3 rd grade homeroom teachers had a higher rate of depressive symptoms than the 1 st and 2nd grade homeroom teachers (probable depression: $39.56 \%$ vs $26.95 \%$, definite depression $17.58 \%$ vs $11.98 \%$ ).

Conclusion This study was the first to investigate the prevalence of depressive symptoms among teachers in Korea. Workers are generally known to have a lower risk of depression than the general population, but teachers have found the opposite result. The resolution of teacher's mental health problems are almost entirely up to the individual teacher. Taking into account the impact of teacher mental health on individuals, students and schools, structural intervention is urgently needed.

\section{PREVALENCE OF AND FACTORS ASSOCIATED WITH BURNOUT AMONG HEALTH CARE PROFESSIONALS IN ARAB COUNTRIES: A SYSTEMATIC REVIEW}

'I Elbarazi, ${ }^{1,2}$ T Loney* ${ }^{3}{ }^{3}$ Yousef, ${ }^{1}$ A Elias. 'Institute of Public Health, College of Medicine and Health Sciences, United Arab Emirates University, Al Ain, PO Box 17666, United Arab Emirates; ${ }^{2}$ Mohammed Bin Rashid University of Medicine and Health Sciences, PO Box 505055, Dubai, United Arab Emirates; ${ }^{3}$ Department of Psychiatry and Behavioural Science, College of Medicine and Health Sciences, United Arab Emirates University, Al Ain, PO Box 17666, United Arab Emirates

\subsection{6/oemed-2018-ICOHabstracts. 1742}

Introduction Burnout among healthcare professionals is one of the key challenges affecting health care practice and quality of care. This systematic review aims:

1. estimate the prevalence of burnout among health care professionals (HCP) in Arab countries; and

2. explore individual and work-related factors associated with burnout in this population.

Methods Multiple electronic databases were searched for studies published in English or Arabic from January 1980 to November 2014 assessing burnout (using the Maslach Burnout Inventory; $\mathrm{MBI}$ ) amongst health care professionals (HCP) in Arab countries.

Results Nineteen studies ( $\mathrm{n}=4108 ; 49.3 \%$ females) conducted on HCP in Bahrain, Egypt, Jordan, Lebanon, Palestine, Saudi Arabia and Yemen were included in this review. There was a wide range of prevalence estimates for the three MBI subscales, high Emotional Exhaustion (20.0\%-81.0\%), high Depersonalization (9.2\%-80.0\%), and low Personal Accomplishment (13.3\%-85.8\%). Gender, nationality, service duration, working hours, and shift patterns were all significantly associated with burnout.

Conclusion Within the constraints of the study and the range of quality papers available, our review revealed moderate-tohigh estimates of self-reported burnout among HCP in Arab countries that are similar to rates in non-Arabic speaking westernised developed countries. In order to develop culturally appropriate interventions, further research using longitudinal designs is needed to confirm the risk factors for burnout in specific HCP settings and specialties in Arab countries.

\section{ASSESSMENT OF PSYCHOSOCIAL RISKS AND WORK ABILITY AMONG SEAFARERS}

${ }^{1} \mathrm{~B}$ Matulić ${ }^{*},{ }^{2} \mathrm{~N}$ Varljen, ${ }^{3} \mathrm{M}$ Matulić, ${ }^{4} \mathrm{M}$ Mustać, ${ }^{2} \mathrm{~A}$ Šimundić, ${ }^{5} \mathrm{M}$ Milošević. ${ }^{1}$ University of Zagreb, School of Medicine, Zagreb, Croatia; ${ }^{2}$ Occupational medicine clinic, Rijeka, Croatia; ${ }^{3}$ Occupational health senvice institute Benedikt, Split, Croatia; ${ }^{4}$ Occupational medicine clinic Mate Mustać, Zadar, Croatia; ${ }^{5}$ University of Zagreb, School of Medicine; WHO Collaborative Centre for Occupational Health, Croatia

\subsection{6/oemed-2018-ICOHabstracts. 1743}

Introduction Work conditions have a great impact on the work ability of a seafarer, whether it is about relationship with superiors, working conditions or job description. Ability to accomplish tasks is also very important sign of wellbeing of a worker. As seafarers face a wide range of psychosocial stressors on board, they may be vulnerable to develop burnout syndrome. The aim of this study was to determine influence of different psychosocial risk factors on seafarers work ability. Methods Field study among seafarers in Croatia was performed from December 2016 until February 2017. Questionnaires regarding burnout, workplace stress levels, work ability index (WAI) and socio-demographic questions have been administered to 222 seafarers in three different cities on the Adriatic coast (Rijeka, Zadar and Split). Response rate was $100 \%$ giving a total number of 222 participants. Statistical analysis was performed with IBM SPSS Statistics version 24 and all $\mathrm{P}$ values below 0.05 were considered significant.

Result All included seafarers were men with average age of 43.11 11.39 years. Thirty-eight seafarers $(17.1 \%)$ had unsatisfactory WAI score $(\leq 36)$ with very high priority of adequate preventive measure in work ability preservation. Pressure at work, uncertainty of work and boredom were most prominent workplace stressors. High burnout level had near $4 \%$ of all participants (8/222) and 51 (22.9\%) had initial stage of burnout. Binary logistic regression showed that higher burnout level is most significant predictor of low work ability $(\mathrm{OR}=11.3 ; 95 \% \mathrm{CI}: 3.3$ to $38.8 ; \mathrm{p}<0.001)$ controlled for age, education level, work tenure, job type and other workplace stressors.

Discussion Results have shown that the prevention of burnout syndrome should be done as soon as possible to maintain adequate work ability. The most vulnerable groups are young and unexperienced workers. Employers should be advised to have appropriate interventions on everyday job routine to prevent boredom especially on long trips.

\section{DISTRESS AND PSYCHOSOCIAL RISK FACTORS IN CHILEAN COPPER MINERS: A CROSS SECTIONAL STUDY}

${ }^{1,2}$ Méndez José* ${ }^{3}$ Carvalho Denise, ${ }^{3}$ Radon Katja, ${ }^{4}$ Silva Helia. ${ }^{1}$ Codelco Chile, División Andina, Los Andes, Chile; '2Sociedad Chilena de Medicina del Trabajo, Santiago, Chile; ${ }^{3}$ Center for International Health, Ludwig-Maximilians-Universität München, Munich, Germany; ${ }^{4}$ Escuela de Salud Pública, Universidad de Chile, Santiago, Chile

10.1136/oemed-2018-ICOHabstracts. 1744 